\title{
Seducing astrocytes to the dark side
}

\author{
Cell Research (2017) 27:726-727. doi:10.1038/cr.2017.37; published online 17 March 2017
}

\begin{abstract}
After injury and in disease of the central nervous system (CNS), local cells called astrocytes respond with diverse molecular changes whose functional consequences are incompletely understood. A combined genomic and experimental analysis shows that classically-activated microglia, which are innate immune cells resident in CNS neural tissue, release molecules that drive astrocytes into a neurotoxic state, raising important questions about potential adaptive and maladaptive functions of such a mechanism.
\end{abstract}

Astrocytes, a type of glial cell found throughout the brain and spinal cord, play indispensable roles in sustaining normal neurologic function and responding to all forms of central nervous system (CNS) injury and disease. Following CNS insults, astrocytes undergo rapid changes in gene expression, morphology and function collectively referred to as astrocyte reactivity. Astrocyte reactivity is not a single all-or-none response, but instead is a diverse continuum of graded and context-specific responses that may result in either adaptive or maladaptive effects $[1,2]$. To begin dissecting and understanding the diversity, regulation and functions of reactive astrocyte responses, several studies have employed quantitative genomic analyses to characterize astrocyte gene expression profiles in various CNS disorders. The results of these studies highlights the existence of molecularly diverse subtypes of reactive astrocytes that may serve a multitude of beneficial or deleterious roles [3-5]. Nevertheless, the identity of key cellular and molecular mechanisms driving such diverse responses, and the functional implications of different forms of as- trocyte reactivity for CNS disorders, remain central questions in the study of the neurobiology of disease.

In a recent study from the Barres group, Liddelow et al. [6] identify a mechanism through which classically activated microglia, innate inflammatory cells that are resident in CNS neural tissue, can drive reactive astrocytes into a neurotoxic state (Figure 1). The authors investigated the regulation and function of two markedly different subtypes of reactive astrocytes previously characterized in the Barres lab by genomic analysis of mouse models of two divergent forms of CNS insult: (i) neuroinflammation due to systemic injection of a bacterial endotoxin, lipopolysaccharide (LPS), and (ii) ischemic brain injury [3]. Neuroinflammation-reactive astrocytes, which in the previous study were found to exhibit a potentially noxious proinflammatory genomic profile [3], were in the present study termed 'A1' [6]. In contrast, ischemia-reactive astrocytes, which exhibit a more neuro-supportive gene profile with enhanced expression of metabolism-associated genes and neurotrophic factors, were termed 'A2'. In their first set of experiments, Liddelow et al. [6] used a combination of in vivo quantitative genomic profiling, genetically modified mice and purified rodent astrocyte cultures, and found that LPS acted indirectly through microglia to induce A1 astrocytes. Indeed, simply treating astrocyte cultures with LPS-stimulated microglia conditioned culture media was sufficient to drive A1-associated gene expression, while direct stimulation of astrocytes with LPS was unable to induce the $\mathrm{A} 1$ reactivity profile.

The authors then conducted a tour de force of trial and error experiments to identify the microglia-derived mediator(s) of the A1 phenotype. By screening numerous combinations of molecules from diverse functional groups for their ability to induce the $\mathrm{A} 1$ reactive gene expression profile in cultured astrocytes, the authors identified the cytokines Interleukin 1 alpha (Il1 $\alpha$ ) and Tumor Necrosis Factor (TNF), in combination with compliment subcomponent $\mathrm{Clq}$, as required and sufficient to recapitulate the $\mathrm{A} 1$ expression profile induced by microglia. Astrocyte transformation to A1 could be rapidly reversed by treatment with Transforming Growth Factor beta (TGF $\beta$ ), but not simply by the removal of A1 mediators I11 $\alpha$, TNF and C1q, suggesting that states of reactivity may endure until counteracted by specific molecular signals in the injury microenvironment. This may be particularly important for understanding persistent astrocyte reactivity in the context of chronic neurological disorders.

Next, using A1 astrocyte cultures, the authors found that multiple well-established astrocyte functions were severely impaired in A1 cells, including the ability to promote structural and functional synapse formation [7, 8], and phagocytose synaptic debris [9]. Remarkably, the loss of various normal functions was accompanied by an unusual gain of function - the ability to selectively kill neurons via an as yet unidentified secreted molecule. Moreover, evidence is provided that the neurotoxic A1 astrocytes can play a central role in mediating the death of damaged neurons following CNS traumatic injury in vivo. Preventing A1 reactive astrocyte formation in response to optic nerve crush injury, either by in 


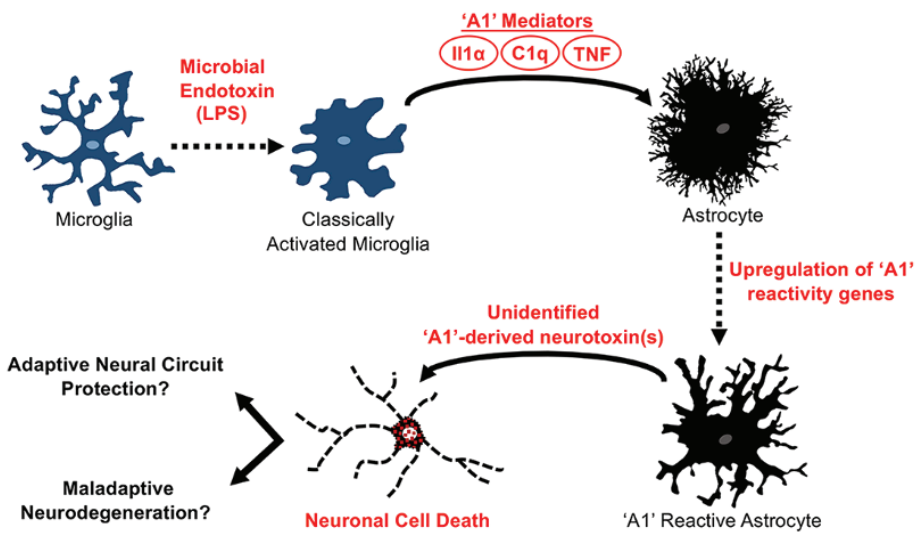

Figure 1 Activated microglia can drive reactive astrocytes into a neurotoxic state. Lipopolysaccharide (LPS), a microbial endotoxin, activates microglia to release Interleukin 1 alpha $(I I \alpha)$, Tumor Necrosis Factor (TNF), and compliment subcomponent $\mathrm{C} 1 \mathrm{q}$. These microglia-derived molecular signals are required and sufficient to drive astrocytes into an ' $\mathrm{A} 1$ ' reactive state characterized by upregulated expression of A1-specific genes and the release of a still unidentified, but potent, toxin that selectively kills neurons. It is not yet clear whether this neurotoxic astrocyte function acts adaptively to remove irreversibly damaged and dysfunctional neurons, or acts maladaptively to remove healthy neurons that are being inappropriately targeted.

vivo administration of Il1 $\alpha$, TNF and $\mathrm{Clq}$ function-blocking antibodies, or using $\mathrm{I} 11 \alpha^{--} \mathrm{TNF}^{--} \mathrm{C} 1 \mathrm{q}$ triple-knockout mice, greatly attenuated the loss of axotomized retinal ganglion neurons. Lastly, using expression of complement component 3 (C3) as a marker, A1 astrocytes were found to be present in tissue sections derived from patients with common neurodegenerative diseases, including Huntington's, Alzheimer's, multiple sclerosis, Parkinson's and ALS, suggesting a potential role for A1 astrocytes in human CNS disorders.

These findings raise important questions. First, what is the identity of the neurotoxin produced by $\mathrm{A} 1$ astrocytes? Work is underway in this regard. Second, what purpose(s) might be served by the existence and evolutionary conservation of a mechanism to generate neurotoxic astrocytes? One possibility could be the adaptive function of removing malfunctioning neurons from neural networks to prevent circuit disruption and neurologic dysfunction after either injury, microbial infection or neurodegenerative disease. Another potential adaptive function might be during neurodevelopment, where naturally occurring cell death removes large numbers of neurons that are either dysfunctional or inappropriately wired [10]. Whether or not A1 astrocytes participate in such mechanisms awaits further study. Interestingly, the observation by Liddelow et al. that $\mathrm{A} 1$ astrocytes mediate the death of axotomized neurons might be consistent with such a role. An equally important third question is to what degree do A1 astrocytes participate in maladaptive neurodegenerative events. Neuronal loss is a feature of many neurodegenerative conditions and the underlying mechanisms are not well understood. The observation by Liddelow et al. that astrocytes expressing the A1 marker $\mathrm{C} 3$ are present in tissue from a variety of human neurodegenerative conditions suggests that $\mathrm{A} 1$ astrocytes may be exerting neurotoxic functions in such conditions. Further work is called for. In particular, it will be interesting and critical to determine whether or not potential A1 astrocyte neurotoxic activities are adaptive or maladaptive. Is A1 function adaptively removing irreversibly damaged or dysfunctional neurons that might disrupt neural circuit function, or is A1 function maladaptively removing healthy neurons that are being inappropriately targeted? Understanding this distinction is critical to determine whether inhibiting A1 astrocyte activity will be beneficial or not.

Lastly, the ground-breaking study by Liddelow et al. underscores the notion that understanding and modulating astrocyte reactivity represents a rich new area for identifying potential therapeutic targets for neurological disorders. In this regard, further research is also warranted to define astrocyte intracellular signaling pathways that drive reactive changes in gene expression and thereby cell function. Transcriptional regulatory molecules ultimately transmit extracellular stimuli into changes in gene expression, positioning them as promising targets to selectively manipulate subtypes of reactive astrocytes across a broad range of CNS disorders.

Joshua E Burda ${ }^{1}$, Michael V Sofroniew ${ }^{1}$

${ }^{I}$ Department of Neurobiology, David Geffen School of Medicine, University of California, Los Angeles, Los Angeles, CA 90095, USA

Correspondence: Joshua E Burda ${ }^{a}$, Michael V Sofroniew ${ }^{\mathrm{b}}$

aE-mail: jburda@mednet.ucla.edu

bE-mail: sofroniew@mednet.ucla.edu

\section{References}

1 Burda JE, Sofroniew MV. Neuron 2014; 81:229-248.

2 Khakh BS, Sofroniew MV. Nat Neurosci 2015; 18:942-952.

3 Zamanian JL, Xu L, Foo LC, et al. J Neurosci 2012; 32:6391-6410.

4 Zhang Y, Sloan SA, Clarke LE, et al. Neuron 2016; 89:37-53.

5 Anderson MA, Burda JE, Ren Y, et al. Nature 2016; 532:195-200.

6 Liddelow SA, Guttenplan KA, Clarke LE, et al. Nature 2017; 541:481-487.

7 Kucukdereli H, Allen NJ, Lee AT, et al. Proc Natl Acad Sci USA 2011; 108:E440-E449.

8 Allen NJ, Bennett ML, Foo LC, et al. Nature 2012; 486:410-414.

9 Chung WS, Clarke LE, Wang GX, et al. $\mathrm{Na}$ ture 2013; 504:394-400.

10 Cowan WM, Fawcett JW, O'Leary DD, et al. Science 1984; 225:1258-1265. 